\title{
Process for Compensating Local Magnetic Perturbations on Ferromagnetic Surfaces
}

\author{
Antonio Villalba Madrid, Alejandro Álvarez Melcón \\ Electromagnetics and Telecommunications Group, Technical University of Cartagena, Cartagena, Spain. \\ Email: anvima@gmail.com; alejandro.alvarez@upct.es
}

Received August $3^{\text {rd }}, 2012$; revised September $7^{\text {th }}, 2012$; accepted September $17^{\text {th }}, 2012$

\begin{abstract}
This paper addresses a practical application for the compensation of a local magnetic perturbation in a ship in the near field region. The process will avoid expensive deperming techniques usually applied to ships to treat magnetic anomalies. The technique includes a new system to construct magnetic maps on flat ferromagnetic surfaces. Once the magnetic maps are obtained, a new system is proposed to evaluate and locate local magnetic perturbations. Once the local perturbations are located, they are compensated by local degaussing coils. The new technique has been applied to the detection of local magnetic perturbations in four naval platforms. Two of them presented important magnetic anomalies, and were successfully detected and corrected by applying the new technique, thus showing its practical value.
\end{abstract}

Keywords: Genetic Algorithms; Magnetic Compensation; Optimization Methods; Magnetic Mapping; Static Magnetic

\section{Introduction}

There are currently two technological trends to assure magnetically silent naval platforms. One option is to invest in complex degaussing systems [1,2] in order to avoid costly deperming processes. However, it is generally assumed that deperming cycles during the life-cycle of a vessel cannot be completely avoided. If a deperming process is applied, then degaussing systems become less important. In any case, the new navigation and detection systems require very low levels of magnetic perturbation on board naval platforms, for their correct operation. These strict requirements cannot be usually fulfilled using either degaussing or deperming systems separately. In most cases a combination of both techniques is needed. If a vessel is compensated by a degaussing system, important local magnetic perturbations may still be present in certain areas of the ship, which may induce strong perturbations on the relevant bearing instrumentation. Generally, it is assumed that available calibration processes cannot eliminate the high local gradients that can have an influence on some sensitive bearing magnetic sensors, like the bearing sensor of a helicopter over a flight deck. In addition, this local behavior cannot be predicted by the magnetic ship models (theoretical or numerical) used in the design of the degaussing system coils [3-6]. This is mainly because the local anomaly is produced by the magnetization history of the material, which is unpredictable. On the other hand, although a deperming process may erase the magnetic behavior of the vessel, it does not guarantee a magnetic compensation over time. Usually this costly process must be repeated periodically during the life of the ship.

In the above context, this paper proposes an alternative technique for eliminating local magnetic perturbations on naval platforms avoiding complex deperming processes. While traditional degaussing systems are designed for compensation of magnetic perturbations in the far-field region, the new system is able to compensate local magnetic perturbations in the near-field region, assuring a correct operation on the sensitive navigation instruments, and at the same time avoiding the costly deperming processes.

The compensation of magnetic anomalies in vessels with a degaussing system is carried out by using a set of coils, carrying steady state uniform currents, which produce magnetic fields opposite to the magnetic anomalies. The values of the currents and the number of turns in each coil can be optimized with different calibration techniques. The authors have proposed in [1] a novel calibration technique based on genetic algorithms $[7,8]$. Other alternative approach successfully used for this optimization problem is the Particle Swarm algorithm [9]. The main principles of magnetic compensations were defined in [10]. In these works it is shown that the new calibration technique was able to reduce not only the absolute value of the magnetic field, but also it could reduce the gradient of the associated magnetic anomalies. This gradient is a key parameter for the correct operation of many modern ocean de- 
tection systems. Usually, degaussing systems perform the magnetic compensation in the far-field, where the vessel tends to behave as an equivalent magnetic dipole [11]. However, this far-field compensation does not guarantee an effective local compensation in areas close to the vessel, which may present important values of the magnetic field and of its gradient. This is mainly because the distance introduces and effect of low-pass filtering on the gradient of the magnetic field [6]. For instance, two magnetic dipoles separated at a distance " $d$ " behave like a single dipole if it is tested at a distance greater than the separation " $d$ ". Therefore, important gradients present in the near-field are filtered out in the far-field [6]. In a standard degaussing compensation system, the magnetic sensors are located at a depth equal to the length of the vessel, and therefore the strong gradients occurring in the near-field region are not detected. Moreover, it does not exist readily available techniques for the compensation of these near-field magnetic anomalies.

Currently, when there is a problem associated to a local magnetic perturbation, the only possible solution is to apply a global deperming process to the naval platform. This is a very costly and complex technique, requiring very specialized laboratories and equipment. This paper solves this problem, by proposing a novel and effective technique for the compensation of local magnetic anomalies without the need to resort to complex deperming processes. The new technique is simple, since it uses currently available degaussing infrastructure, and is based on three main steps:

- Measurement of near-field magnetic maps on the deck of the naval platform. For this task we propose a novel experimental facility, which allows to perform the measurements in a semi-automatic fashion.

- Once the magnetic maps of the naval platform deck are available, we propose a novel post-processing technique to identify the exact location of the main magnetic perturbations. This step is based on the definition of new relevant parameters which are used to detect the location and to quantify the severity of the magnetic perturbation.

- Once the area suffering from the magnetic anomaly has been identified, we propose two different techniques for the local compensation. The techniques are applied depending on the nature of the magnetic anomaly detected, namely:

- Anomaly produced by well identified sources: in this case we propose to compensate the anomaly directly acting on the magnetic source producing the anomaly. This can be done with additional degaussing coils placed around the source. The new coils will tend to annihilate the magnetic field produced by the source. In this way magnetic compensation is achieved in the whole area of the platform deck.
- If the source of the magnetic anomaly is produced by several distributed sources, we propose to perform a magnetic compensation localized in the critical sensitive area on the deck, where operation of the on-board sensors must be assured. In this case the goal is to achieve a magnetic compensation in these sensitive areas of the deck.

The new procedure has been used in the study of four identical naval platforms, and in one additional platform selected as reference. The reference platform is known to be free of magnetic anomalies, and it is used to compute the threshold levels of the different parameters used to detect magnetic anomalies (step 2). When the relevant parameters are under the threshold levels, the naval platform operates in a save condition, with no interference with the on-board magnetic sensors.

The four platforms are all equal, but they present different magnetic anomalies because they have been exposed to different magnetic histories. In view on the magnitude and on the source of the magnetic anomalies, we have classified the four naval platforms into three different categories:

- Naval platform with small magnetic anomalies, which does not require magnetic compensation.

- Anomaly of type 1: Strong anomaly with well identified source.

- Anomaly of type 2: Medium anomaly produced by several distributed sources (several elements) of the naval platform.

In this paper, after the description of the novel proposed technique, we present the results obtained for these 4 naval platforms, including the improvements achieved in their magnetic compensation. Results show that the new proposed technique is indeed efficient to treat local magnetic anomalies, avoiding lengthy and costly deperming processes.

\section{Description of the Magnetic Compensation System}

As already indicated, the new technique is composed of three different steps. In the first step we propose a new system for the semi-automatic measurement of magnetic maps on the deck of naval platforms. The second technique introduces new parameters which are used to locate and to quantify the magnetic anomalies. Finally, in the third step the magnetic compensation is effectively applied depending on the type of anomaly detected. In the next sub-sections we describe all three different steps.

\subsection{Multisensor Platform for the Semi-Automatic Measurement of Magnetic Maps}

The new system for the measurement of magnetic maps on the deck of naval platforms is composed of a mobile 
trolley made from non-magnetic material, containing three magnetic sensors and several electronic equipments, as shown in Figure 1.

The magnetic field is captured with full tri-axial magnetic sensors, and a two-axis tilt-meter is used to measure the pitch and roll associated to each measured point. The pitch and roll information is used to compensate for the natural oscillations in the deck during the measurement of the magnetic field at each point of the deck by the magnetic sensors. The presence of these oscillations is due to the natural movement of the naval platform and to the roughness of the deck surface.

For the measurement of the magnetic map an origin is selected on the deck. Starting from the origin, the trolley moves by columns capturing the magnetic field in a mesh of points. The distance of the points in the deck to form the rows of the mesh is adjusted with a digital encoder. The encoder is fixed to the axis of one wheel in the trolley, and gives the angle of rotation encoded in binary form. With this information and with the radius of the wheel, it is possible to compute the distance between two consecutive points in each row of the mapping system. All these data is captured by a sampling card connected to a computer through a USB port. The computer has specific software, developed in LabView, for the storage and processing of the received information. The graphic interface of the software is shown in Figure 2. The whole system is completed with batteries for autonomous operation during two hours, and can be seen in Figure 1.

Before measurements are taken, the system needs to be calibrated in distance. This is done by selecting a given distance on the deck. This information is passed to the graphic interface of Figure 2, and then the trolley is made to cover the specified distance. The calibration process measures the total number of pulses of the digital encoder in the specified distance. With this information it computes the number of pulses of the digital encoder per

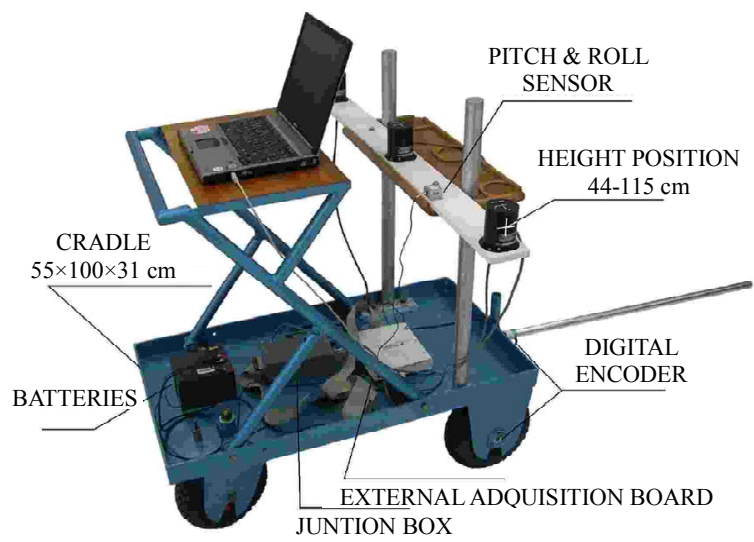

Figure 1. Mobile trolley with magnetic sensors and other electronic equipment used for the measurement of magnetic maps on the deck of naval platforms.

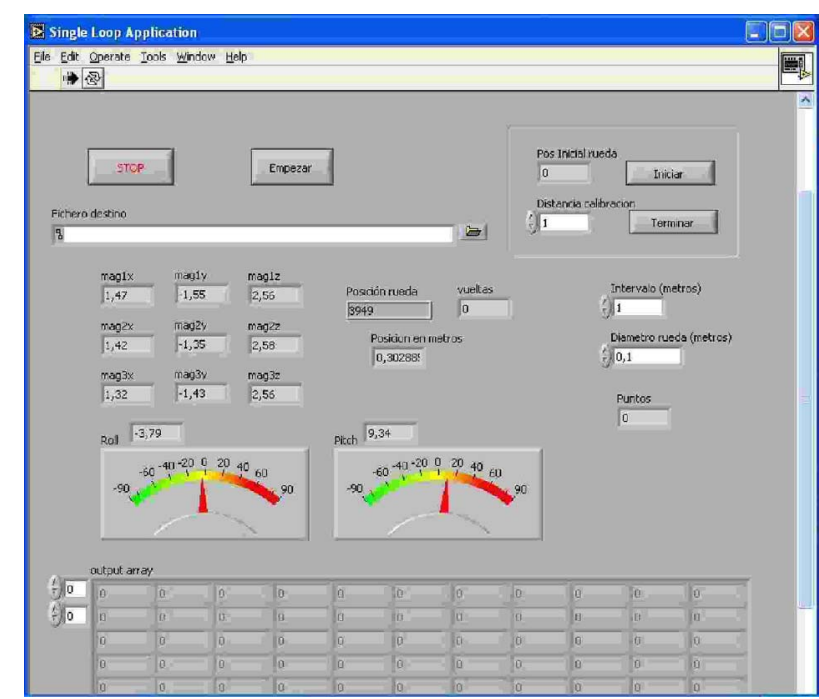

Figure 2. Graphic interface of the software developed for capturing and processing of the information.

unit length. Once the system is calibrated it is possible to encoder in the specified distance. With this information it computes the number of pulses of the digital encoder per adjust the number of sampling points per unit length, therefore effectively selecting the mesh density.

During the measurement procedure, the signals obtained by the different sensors (magnetic sensors, digital encoder and tilt-meter) are captured by the sampling card and are displayed in the graphic interface (Figure 2). The system allows capturing simultaneously three columns of the mesh, since the trolley is provided with three equally spaced magnetic sensors, as shown in Figure 1. In this way the measurement time is consequently reduced by three, until the whole mesh is covered with the system.

In a practical situation, the raw data measured by the system need to be transformed before it can be used for the detection of magnetic anomalies. The first transformation is needed to correct the pitch and roll of the naval platform at each sample point. With the information provided by the tilt-meter, the magnetic field measured at each point is corrected with the following matrix transformation:

$$
\begin{aligned}
& \left(\begin{array}{l}
B_{x}\left(i, j, z_{0}\right) \\
B_{y}\left(i, j, z_{0}\right) \\
B_{z}\left(i, j, z_{0}\right)
\end{array}\right) \\
& =\left(\begin{array}{ccc}
\cos \beta & \sin \alpha \cdot \sin \beta & \sin \beta \cdot \cos \alpha \\
0 & \cos \alpha & -\sin \alpha \\
-\sin \beta & \sin \alpha \cdot \cos \beta & \cos \alpha \cdot \cos \beta
\end{array}\right) \cdot\left(\begin{array}{c}
B_{x}^{\prime}\left(i, j, z_{0}\right) \\
B_{y}^{\prime}\left(i, j, z_{0}\right) \\
B_{z}^{\prime}\left(i, j, z_{0}\right)
\end{array}\right)
\end{aligned}
$$

where $B_{x}^{\prime}\left(i, j, z_{0}\right), B_{y}^{\prime}\left(i, j, z_{0}\right)$ and $B_{z}^{\prime}\left(i, j, z_{0}\right)$ are the measured components of the magnetic field at point $(i, j)$ of the mesh, taken at a fixed height $z_{0}$ above the deck surface. The angles $\alpha$ and $\beta$ correspond to the measured roll and 
pitch angles at each point, respectively, and $B_{x}\left(i, j, z_{0}\right), B_{y}$ $\left(i, j, z_{0}\right)$ and $B_{z}\left(i, j, z_{0}\right)$ are the corresponding components of the corrected magnetic field.

Another useful transformation may be applied to the measured raw data, to align the selected spatial reference system with the magnetic reference system. This is useful in many practical situations, when the orientation of the sensors in the trolley does not coincide with the selected spatial reference system. For instance, if the $x$ - and $y$-axis of the magnetic sensors are inverted with respect to the spatial reference system, the following transformation will be applied:

$$
\left(\begin{array}{l}
B_{x}^{\prime \prime}\left(i, j, z_{0}\right) \\
B_{y}^{\prime \prime}\left(i, j, z_{0}\right) \\
B_{z}^{\prime \prime}\left(i, j, z_{0}\right)
\end{array}\right)=\left(\begin{array}{ccc}
-1 & 0 & 0 \\
0 & 1 & 0 \\
0 & 0 & -1
\end{array}\right) \cdot\left(\begin{array}{l}
B_{x}\left(i, j, z_{0}\right) \\
B_{y}\left(i, j, z_{0}\right) \\
B_{z}\left(i, j, z_{0}\right)
\end{array}\right)
$$

The above concept can be generalized to cover other practical situations, when the measurements cannot be captured sequentially due to contingent operational reasons. In this case, the measurements are taken in any prescribed order, and a matrix transformation is then applied to re-order the captured data into the correct sequence of rows and columns of the mesh. For instance, consider an example where measurements need to be started in column 30 and finish in column 1. In this case, the following matrix transformation is applied:

$$
\begin{aligned}
& \left(\begin{array}{cccccc}
P_{1,1} & P_{1,2} & \cdots & \cdots & P_{1,29} & P_{1,30} \\
P_{2,1} & P_{2,2} & \cdots & \cdots & P_{2,29} & P_{2,30} \\
\vdots & \vdots & \vdots & \vdots & \vdots & \vdots \\
P_{23,1} & P_{23,2} & \cdots & \cdots & P_{23,29} & P_{23,30} \\
P_{24,1} & P_{24,2} & \cdots & \cdots & P_{24,29} & P_{24,30}
\end{array}\right) \\
& =\left(\begin{array}{cccccc}
P_{1,30} & P_{1,29} & \cdots & \cdots & P_{1,2} & P_{1,1} \\
P_{2,30} & P_{2,29} & \cdots & \cdots & P_{2,2} & P_{2,1} \\
\vdots & \vdots & \vdots & \vdots & \vdots & \vdots \\
P_{23,30} & P_{23,29} & \cdots & \cdots & P_{23,2} & P_{23,1} \\
P_{24,30} & P_{24,29} & \cdots & \cdots & P_{24,2} & P_{24,1}
\end{array}\right) \\
& \times\left(\begin{array}{cccccc}
0 & 0 & \cdots & \cdots & 0 & 1 \\
0 & 0 & \cdots & \cdots & 1 & 0 \\
\vdots & \vdots & \vdots & \vdots & \vdots & \vdots \\
0 & 1 & \cdots & \cdots & 0 & 0 \\
1 & 0 & \cdots & \cdots & 0 & 0
\end{array}\right)
\end{aligned}
$$

where $P$ is the matrix containing the raw data in the measured order, and $P$ is the transformed matrix in the correct final order. In general, if the $n$-measured column corresponds to the $m$-column of the final matrix, a " 1 " will be added at the position $(m, n)$ of the above transformation matrix.

Finally, a last transformation is needed to convert the sampling points of the mesh into real spatial points, which are mapped onto the deck of the naval platform. This last transformation is performed as:

$$
\left(\begin{array}{l}
r_{x} \\
r_{y} \\
r_{z}
\end{array}\right)=\left(\begin{array}{ccc}
\frac{2 \pi \cdot r}{2^{n_{\mathrm{bits}}}} & 0 & 0 \\
0 & D y & 0 \\
0 & 0 & 1
\end{array}\right) \cdot\left(\begin{array}{l}
n_{x} \\
n_{y} \\
n_{z}
\end{array}\right),
$$

where $n_{x}$ is the number of pulses of the digital encoder, $n_{y}$ is the number of sampling columns, and $n_{z}$ is the fixed height above the deck where measurements are taken. Also, $D y$ is the real distance between the columns of the mesh in meters, $r$ is the radius of the trolley wheels, and $\left(r_{x}, r_{y}, r_{z}\right)$ are the final coordinates of the points of the mesh mapped onto the deck of the vessel (in meters). This transformation is useful, for instance, to locate the exact coordinates of the area in the deck affected by a possible magnetic anomaly.

To illustrate the procedure described, we present in Figure 3 the transverse and the vertical components of the magnetic field measured in the first naval platform investigated in our study. Similar magnetic maps are obtained for all other components of the magnetic field without extra effort, since the sensors employed are triaxial. These magnetic maps will serve as the starting point to assess the magnetic anomalies in each naval platform.

\subsection{Assessment and Detection of Magnetic Anomalies}

Once the magnetic map of the vessel is obtained following the previous procedure, the data must be processed in order to detect possible magnetic anomalies. To detect the anomalies, the magnetic maps will be processed by rows and columns independently, and for the three components of the measured magnetic field. Also, it is convenient not to process the whole data corresponding to the total deck area. It is more efficient to process only the

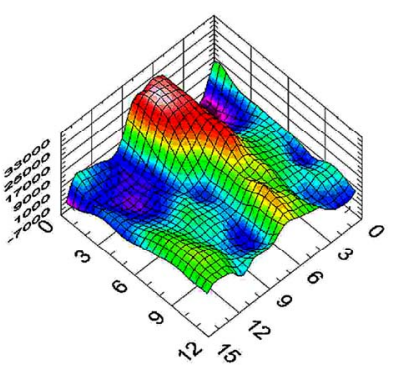

(a)

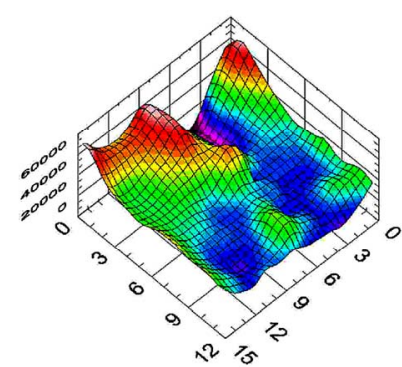

(b)
Figure 3. Measured $x$-component (transversal)-(a) and $z$ component (vertical)-(b), of the magnetic field in the first platform investigated in this study, obtained with the new measurements setup. 
data of sensitive areas, where interference with on-board sensors are critical. For the purpose of our investigation, the whole deck surface is divided into 4 spatial regions $\left(Z_{1}, Z_{2}, Z_{3}\right.$ and $Z_{4}$, Figure 4). From these 4 zones, the $Z_{1}$ and $Z_{2}$ areas present the edge effects of the platform, and they are not sensitive areas for the on-board sensors. The zone $Z_{4}$ is very small, and it is contained inside zone $Z_{3}$. Therefore, the study has been restricted to zone $Z_{3}$ (delimited by points $(8,4),(23,4),(23,12)$ and $(8,12)$ of the magnetic map), where on-board sensor may operate in close vicinity. In Figure 4 we also show the mesh used for the sampling of the magnetic field in the deck area.

The proposed technique for the detection of magnetic anomalies is based on the definition of new parameters, and on the investigation on how these parameters evolve in the area of study. The following parameters are proposed to detect possible magnetic anomalies, using the row and column averages of the measured magnetic maps:

- Variation of the maximum or minimum in nanoTeslas from the average (DM). When the average is taken by rows and for the $x$-component of the magnetic field, the maximum or minimum variation will be called $D M P R X$; when the parameter is computed for the column of the same component the name will be $D M P C X$. Similar parameters are extracted for the other magnetic components, and along the rows and columns of the measured magnetic map.

This parameter is computed with the following expression:

$$
\operatorname{DMPR}(x, y, z)=\operatorname{Max}(\operatorname{MaPR}(x, y, z) ; \operatorname{MiPR}(x, y, z)) .
$$

where $\operatorname{MaPR}(x, y, z)$ and $\operatorname{MiPR}(x, y, z)$ are calculated as,

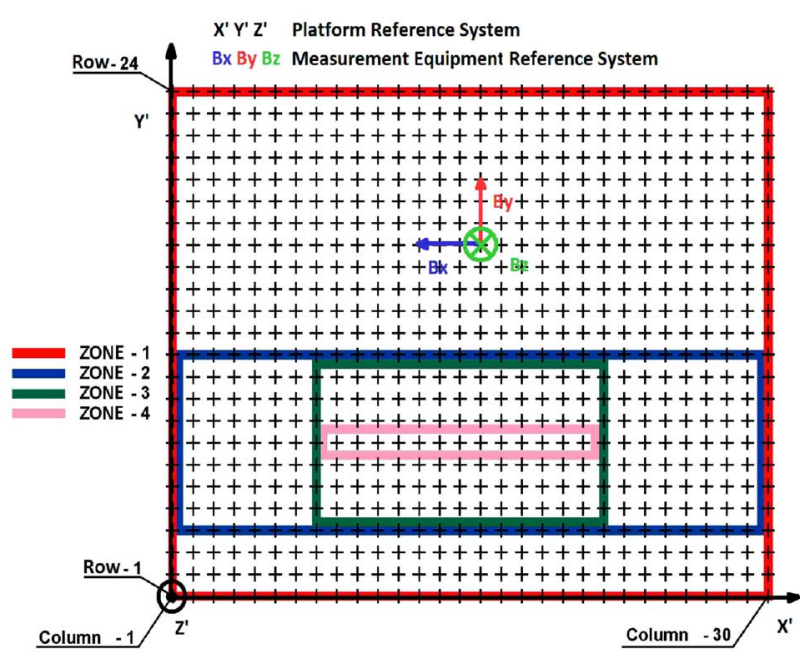

Figure 4. Subdivision of the vessel deck into four spatial zones, and mesh of points used for the magnetic map measurements and reference system.

$$
\begin{aligned}
& \operatorname{MaPR}(x, y, z) \\
= & A B S\left(\operatorname{Max}\left(\left.\operatorname{PR}(i, x, y, z)\right|_{1 \leq i \leq 30}\right)-\frac{1}{30} \sum_{i=1}^{30} \operatorname{PR}(i, x, y, z)\right) \\
& \operatorname{MiPR}(x, y, z) \\
= & A B S\left(\frac{1}{30} \sum_{i=1}^{30} \operatorname{PR}(i, x, y, z)-\operatorname{Min}\left(\left.\operatorname{PR}(i, x, y, z)\right|_{1 \leq i \leq 30}\right)\right)
\end{aligned}
$$

$M a P R$ is the absolute value of the difference between the maximum of averaged magnetic flux density curve of rows in the zone $Z_{3}$ and its averaged value in all the columns of the vessel deck (the value of 30 is the number of columns in the whole vessel deck). The parameter $M i P R$ is calculated in a similar way but for the minimum. The parameter $P R$ is obtained through an average of the magnetic flux density in rows covering the zone $Z_{3}$ of the deck.

$$
\left.P R(i, x, y, z)\right|_{Z_{3}}=\left.\frac{1}{9} \sum_{j=4}^{12}\left(\vec{B}_{P(i, j)}^{H, \theta}(x, y, z)\right)\right|_{\rightarrow^{H, \theta}}
$$

In the last equation, $B_{P(i, j)}(x, y, z)$ is the magnetic flux density of a point $P(i, j)$ of the platform in a given area $H$ of the Earth with heading $\theta$. It can be seen that $P R$ $(i, x, y, z)$ is the magnetic flux density averaged, from row 4 up to row 12 (9 rows), covering all rows in the zone $Z_{3}$ (Figure 4). However, this calculation is done for all 30 columns of the vessel deck, and not only for the columns inside the zone $Z_{3}$. This is needed in order to consider all the information available along the width of the deck when computing the maximum and the minimum values.

The average per columns is calculated in a similar way, but interchanging the columns " $i$ " and rows " $j$ " indexes, obtaining,

$$
\operatorname{DMPR}(x, y, z)=\operatorname{Max}(\operatorname{MaPR}(x, y, z) ; \operatorname{MiPR}(x, y, z)) .
$$

where,

$$
\begin{aligned}
& \operatorname{MaPC}(x, y, z) \\
= & A B S\left(\operatorname{Max}\left(\left.P C(j, x, y, z)\right|_{1 \leq j \leq 24}\right)-\frac{1}{24} \sum_{j=1}^{24} P C(j, x, y, z)\right) \\
& \operatorname{MiPC}(x, y, z) \\
= & A B S\left(\frac{1}{24} \sum_{j=1}^{24} P C(j, x, y, z)-\operatorname{Min}\left(\left.P C(j, x, y, z)\right|_{1 \leq j \leq 24}\right)\right) \\
& \left.P C(j, x, y, z)\right|_{Z_{3}}=\left.\frac{1}{16} \sum_{i=8}^{23}\left(\vec{B}_{P(i, j)}^{H, \theta}(x, y, z)\right)\right|_{1 \leq j \leq 24}
\end{aligned}
$$

In this case, 24 is the number of rows in the whole vessel deck. The columns used to extract $P C(j, x, y, z)$ are 
from column 8 up to column 23 (16 columns), covering all columns in the zone $Z_{3}$ (Figure 4). As before, the calculation is performed for all rows in the vessel deck $(1 \leq$ $j \leq 24$ ), and not only for the rows inside the zone $Z_{3}$. Again, this is needed in order to consider all information available along the length of the deck when calculating the maximum and minimum values.

- Extended Gradient (GE) in nanoTeslas per meter $(\mathrm{nT} / \mathrm{m})$ : it is extracted from the row and column averages, by taking the difference between the maximum and minimum and dividing by the distance between these two points. This parameter, when working per rows and for the $x$-component of the magnetic field will be called GEPRX; when working per columns it will be named GEPCX. This parameter is also extracted for all the components of the magnetic field, and along the rows and columns of the measured magnetic map.

This parameter is computed using the following expressions, for the $x, y$ and $z$-components,

$$
\begin{aligned}
G E P R X & =\frac{1}{\delta_{C} \cdot\left(i x_{\max }-i x_{\min }\right)} \\
\times & {\left[\left.P R\left(i x_{\max }, x\right)\right|_{Z_{3}}-\left.P R\left(i x_{\min }, x\right)\right|_{z_{3}}\right] } \\
G E P R Y= & \frac{1}{\delta_{C} \cdot\left(i y_{\max }-i y_{\min }\right)} \\
\times & \times\left[\left.P R\left(i y_{\max }, y\right)\right|_{Z_{3}}-\left.P R\left(i y_{\min }, y\right)\right|_{Z_{3}}\right] \\
G E P R Z= & \frac{1}{\delta_{C} \cdot\left(i z_{\max }-i z_{\min }\right)} \\
& \times\left[\left.P R\left(i z_{\max }, z\right)\right|_{Z_{3}}-\left.P R\left(i z_{\min }, z\right)\right|_{Z_{3}}\right]
\end{aligned}
$$

Where $\left.P R\left(i x_{\max }, x\right)\right|_{Z_{3}}$, is the maximum, inside the zone $Z_{3}$, of the $\left.P R\left(i x_{\min }, x\right)\right|_{Z_{3}} x$-component of the average $P R$ as defined in Equation (7), is the minimum, and ixmax is the index where the maximum value takes place. When it is multiplied by the distance between columns $\delta_{C}$, the $x$-coordinate in meters is obtained. The same is indicated with $i x \min$ to locate the minimum. Similar notation is used for the calculation of the $y$ - and $z$-components, and for the calculation of the gradient along the columns. In this case we can directly express,

$$
\begin{aligned}
G E P R X= & \frac{1}{\delta_{R} \cdot\left(j x_{\max }-j x_{\min }\right)} \\
\times & {\left[\left.P C\left(i x_{\max }, x\right)\right|_{z_{3}}\right.} \\
& \left.\quad-\left.P C\left(i x_{\min }, x\right)\right|_{z_{3}}\right]
\end{aligned}
$$

$$
\begin{aligned}
G E P C Y & =\frac{1}{\delta_{R} \cdot\left(j y_{\max }-j y_{\min }\right)} \\
& \times\left[\left.P C\left(i y_{\max }, y\right)\right|_{Z_{3}}-\left.P C\left(i y_{\min }, y\right)\right|_{Z_{3}}\right] \\
G E P C Z & =\frac{1}{\delta_{R} \cdot\left(j z_{\max }-j z_{\min }\right)} \\
& \times\left[\left.P C\left(i z_{\max }, z\right)\right|_{Z_{3}}-\left.P C\left(i z_{\min }, z\right)\right|_{Z_{3}}\right]
\end{aligned}
$$

where the average per columns $P C$ is defined in Equation (10), and $\delta_{R}$ is the distance between rows in meters.

- Declination error gradient (GMER): using the row and column average of the $x$ - and $y$-components of the magnetic field, the maximum absolute value of the heading error of the ship is calculated in degrees per meter $(\% / \mathrm{m})$. In the case of rows average the parameter will be called GMERPR, while for columns it is called GMERPC.

This parameter is calculated using the following expression,

$$
\begin{aligned}
& \text { GMERPR } \\
& =\frac{\operatorname{GERPR}\left(i_{\text {max }}\right)-\operatorname{GERPR}\left(i_{\text {min }}\right)}{\delta_{C} \cdot\left(i_{\text {max }}-i_{\text {min }}\right)} ; i_{\text {max }, \text { min }} \in Z_{3}
\end{aligned}
$$

where we have defined,

$$
\begin{aligned}
G E R P R(i)=A B S \times & {\left[\frac{180^{\circ}}{\pi} \times \operatorname{arctg}\left(\frac{P R(i, x)}{P R(i, y)}\right)\right.} \\
& \left.+360^{\circ}-(R G e o-D e c)\right]
\end{aligned}
$$

The calculation of the parameter along columns follows a similar notation, namely,

$$
\begin{aligned}
& \operatorname{GMERPC}=\frac{\operatorname{GERPC}\left(j_{\max }\right)-\operatorname{GERPC}\left(j_{\min }\right)}{\delta_{F} \cdot\left(j_{\max }-j_{\min }\right)} ; \\
& j_{\max , \min } \in Z_{3} \begin{array}{r}
\operatorname{GERPC}(j)=A B S\left[\frac{180^{\circ}}{\pi} \times \operatorname{arctg}\left(\frac{P C(j, x)}{P C(j, y)}\right)\right. \\
\left.+360^{\circ}-(R G e o-D e c)\right]
\end{array}
\end{aligned}
$$

where, in above equations, $P R(i, x)$ is the $x$-component of the averaged $P R$ as defined in Equation (7), and $P R(i, y)$ is a similar quantity but for the $y$-component. In the same way, $P C(j, x)$ is the $x$-component averaged by columns as defined in Equation (10), and $P C(j, y)$ is the same but for the $y$-component. Finally, RGeo is the geographic heading and Dec is the magnetic declination.

For the two first parameters (DM and GE) we need a reference value (REF), which will be used to adjust the threshold to decide the type of anomaly. The reference value (REF) will be measured over the reference platform, for which we know that no anomalies are present. 
For the GMER parameter there is not a reference value, since it measures the real heading error of the ship. The value of DM referred to REF will classify the anomaly as type 2 when it is in the range between $110 \%$ and $200 \%$, and of type 1 when it is above $200 \%$. When this value is below $110 \%$, the anomaly will be considered as not significant. For the GE parameter, again referred to the REF value, differences below $150 \%$ are not important. In the range between $150 \%$ and $250 \%$ will be classified as a type 2 anomaly, and when it is above $250 \%$ the anomaly is considered to be of type 1 .

We have observed that the parameters GE and DM using the rows or columns average have similar behavior. However in the case of GMER there exists an important difference when working by rows or by columns. This is because the GMER parameter measures real errors of heading, and they can be very different in the two main directions of the vessel. When GMERPR is between $0 \% \mathrm{~m}$ and $13 \%$ it is not considered as magnetic anomaly. A type 2 anomaly has a value between $13 \% \mathrm{~m}$ and $18 \%$ m. Values above $18^{\circ} / \mathrm{m}$ indicate that a type 1 anomaly is present. When working by columns, the parameter GMERPC need to be more restrictive, since along this direction there are no symmetries in the ferromagnetic materials of the vessel. As a consequence the parameter is naturally lower, and a lower threshold will indicate the beginning of a magnetic anomaly. In this case, values of GMERPC below $7 \% \mathrm{~m}$ show no anomaly; between $7^{\circ} / \mathrm{m}$ and $10 \%$ an anomaly of type 2 and above $10 \% \mathrm{~m}$ reveals an anomaly of type 1. In Table 1 we collect the classification of the different types of anomaly, depending on the values of the different parameters defined in this work. The thresholds have been obtained from experimentation, taking into consideration the expertise and recommendations of renowned operators in vessels decks. In principle they can be applied to other vessels and platform types.

\subsection{Anomaly Compensation}

Once a given magnetic anomaly is detected using the new parameters defined in the previous section, two methods are suggested to compensate for the magnetic

Table 1. Classification of the different types of anomalies as a function of the different parameters defined in this work.

\begin{tabular}{cccc}
\hline Parameter/ & \multicolumn{3}{c}{ ANOMALY } \\
\cline { 2 - 4 } Reference & $\mathrm{NO}$ & Type 2 & Type 1 \\
\hline $\mathrm{DM} / \mathrm{REF}$ & $<1.1$ & $1.1 \leq \mathrm{DM} / \mathrm{REF}<2$ & $\geq 2$ \\
$\mathrm{GE} / \mathrm{REF}$ & $<1.5$ & $1.5 \leq G E / R E F<2.5$ & $\geq 2.5$ \\
GMERPR & $0<G M E R P R<13$ & $13 \leq G M E R P R<18$ & $G M E R P R \geq 18$ \\
GMERPC & $0<G M E R P C<7$ & $7 \leq G M E R P C<10$ & $G M E R P C \geq 10$ \\
\hline
\end{tabular}

anomaly. In the first case (strong anomaly or type 1) the magnetic source is known and it has been identified. In this situation we propose to use additional coils around the source, to compensate the produced magnetic field directly on the source. The situation of the coils will depend on the orientation of the magnetization created by the source. The second situation is produced by a type 2 anomaly, which is not localized into a specific magnetic source, but rather is due to several distributed elements. In this case we propose to focus the compensation to a given area of the deck, where the sensitive magnetic sensors must operate. This problem will be treated using vertical coils (horizontal plane), with maximum dimensions similar to the distance to the area where the compensation is desired.

For both techniques, the number of coils will depend on the surface to compensate. Also, the compensation of the distributed anomaly (type 2) needs more coils than for anomalies of type 1 . The value of the currents and the number of turns in each coil, in both cases, will be calculated using the Genetic Algorithm (GA) method described in [1].

\section{Results}

The techniques described above have been applied to five naval platforms, one of them taken as reference. As a result of this study we have found a magnetic anomaly in two of them, one of type 1 and the other of type 2 .

As we described in Section 2.2 the detection of the anomalies is based on the study of several parameters. In particular, we have studied DM, and GE for the $x-, y$ - and $z$-components of the magnetic field, evaluated per row and column averages. In addition, the parameter GMER was also evaluated per rows and columns to assess the declination error gradient in both navigation directions of the vessel. In this experiment, the anomalies detected presented strong transversal magnetic components produced by one or several transversal sources. These magnetic anomalies are detected in the $x$-component of the above mentioned parameters. Therefore, we only present the results obtained for the parameters DMPRX, GEPRX, GMERPR and GMERPC.

Figure 5 shows the average of the $x$-component of the magnetic field computed per rows, along the columns defined inside the zone $Z_{3}$ of Figure 4, and for the five naval platforms investigated.

From this average we can easily compute the parameters $D M P R X(\mathrm{nT})$ and $G E P R X(\mathrm{nT} / \mathrm{m})$ for all five naval platforms, as shown in Table 2. From the results we observe that the first naval platform (P1) presents very high values of the DMPRX parameter, indicating a strong anomaly produced by a transversal source. Also the parameter GEPRX is high, confirming the presence of an 

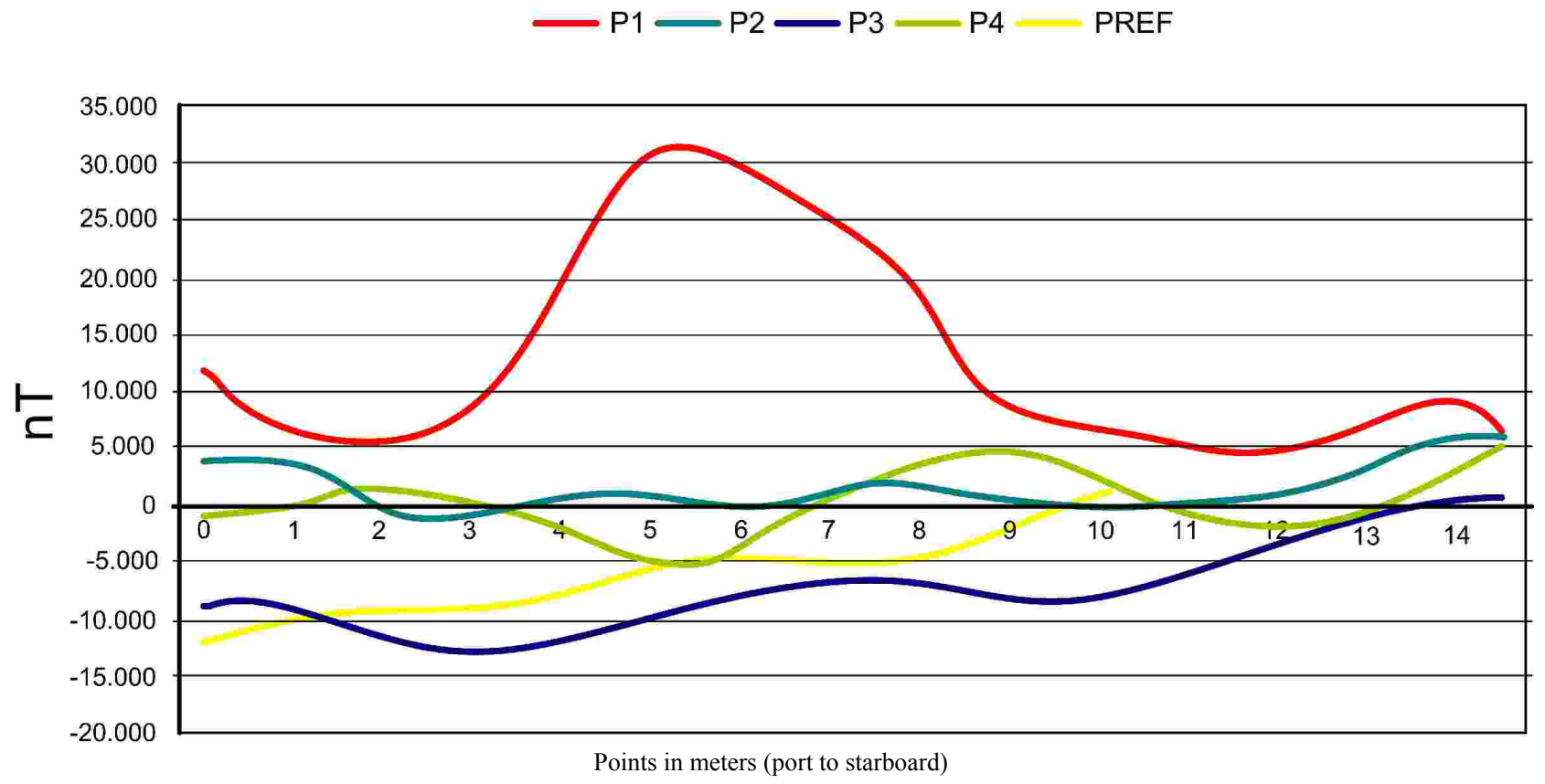

Figure 5. Results obtained for the average of the $x$-component of the magnetic field computed per rows, for all naval platforms studied. Variation along columns inside zone $Z_{3}$.

Table 2. Numerical values obtained for $D M P R X$ and GEPRX for all five naval platforms.

\begin{tabular}{ccccc}
\hline PLATFORM & $\begin{array}{c}\text { DMPRX } \\
(\mathrm{nT})\end{array}$ & DMPRX/REF & $\begin{array}{c}\text { GEPRX } \\
(\mathrm{nT} / \mathrm{m})\end{array}$ & GEPRX/REF \\
\hline PREF & 4.642 & - & 1.245 & - \\
P1 & 18.479 & 3.98 & 4.827 & 3.88 \\
P2 & 1.312 & 0.28 & 1.480 & 1.19 \\
P3 & 4.216 & 0.91 & 855 & 0.69 \\
P4 & 5.207 & 1.21 & 2.467 & 1.98 \\
\hline
\end{tabular}

important anomaly in this platform.

According to the thresholds established in the previous section, we can easily verify that platform (P1) is classified as severe anomaly of type 1 , while platform (P4) as anomaly of type 2. Platforms (P2) and (P3) maintain the $D M P R X$ and GEPRX values below the thresholds $(1.1 \times$ $4.642=5.106$ and $1.5 \times 1.245=1.868$, respectively), and they are thus considered to be free of anomalies.

Once the previous parameters are studied for all three components of the magnetic field, we proceed to the calculation of the heading errors along both rows and columns (GMERPR and GMERPC). These two parameters are extracted from the declination of the horizontal components of the magnetic field (angles between the $x$ and $y$-components of the magnetic field referred to the two main directions of the platform). The variation of the declination along the two main directions inside the zone $Z_{3}$ in all five platforms is shown in Figures 6 and $\mathbf{7}$.

From these Graphics we can extract the final GMERPR and GMERPC parameters for the five platforms studied, as shown in Table 3.

From the obtained results we can conclude that the heading errors are higher in platform (P1) than in the other platforms measured, confirming the strong anomaly present in this platform. According to the thresholds given in the previous section, platform (P1) has again an anomaly of type 1 and platform (P4) has and anomaly of type 2 . These values confirm that the other platforms do not have any magnetic anomaly, since these parameters remain below the specified thresholds.

Once the anomalies have been detected, we propose two different techniques for compensation, depending on the type of the anomaly detected. In the case of type 1 anomaly, present in platform (P1), we propose a compensation acting directly on the magnetic source responsible for the anomaly. For this platform (P1), the critical area is delimited by rows $2-10$ and by columns $9-21$ of the magnetic map. This can be observed in Figure 8, where we show the measured $x$-component of the magnetic field from port to starboard.

From the measured results we can observe both high values and fast variations of this component of the magnetic field.

The compensation in this case is performed with two vertical coils (that we call $\mathrm{MBr}$ and $\mathrm{MEr}$ ) placed under the deck (at a depth of three meters), around the identified 

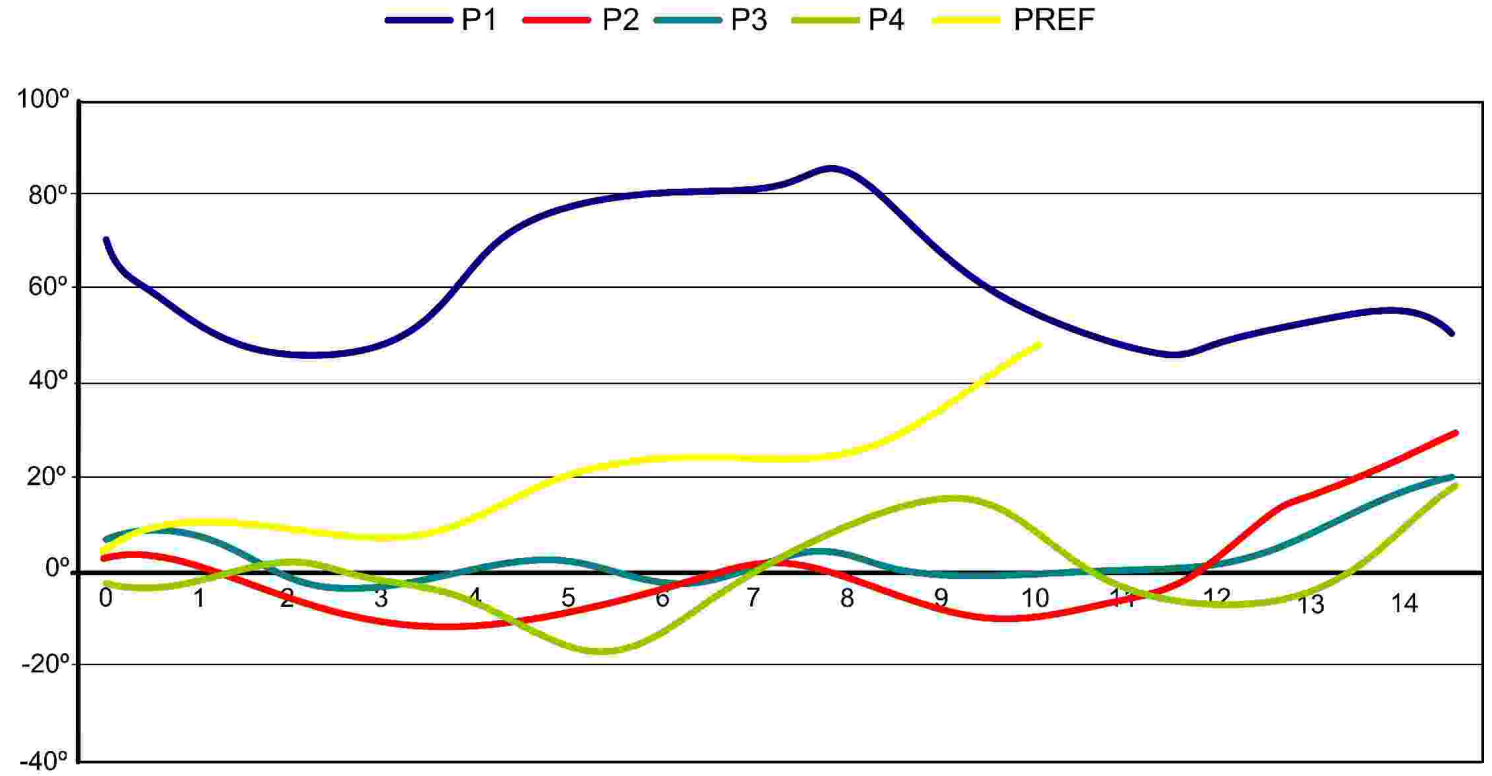

Points in meters (port to starboard)

Figure 6. Absolute declination of the horizontal magnetic field along columns inside zone $Z_{3}$.

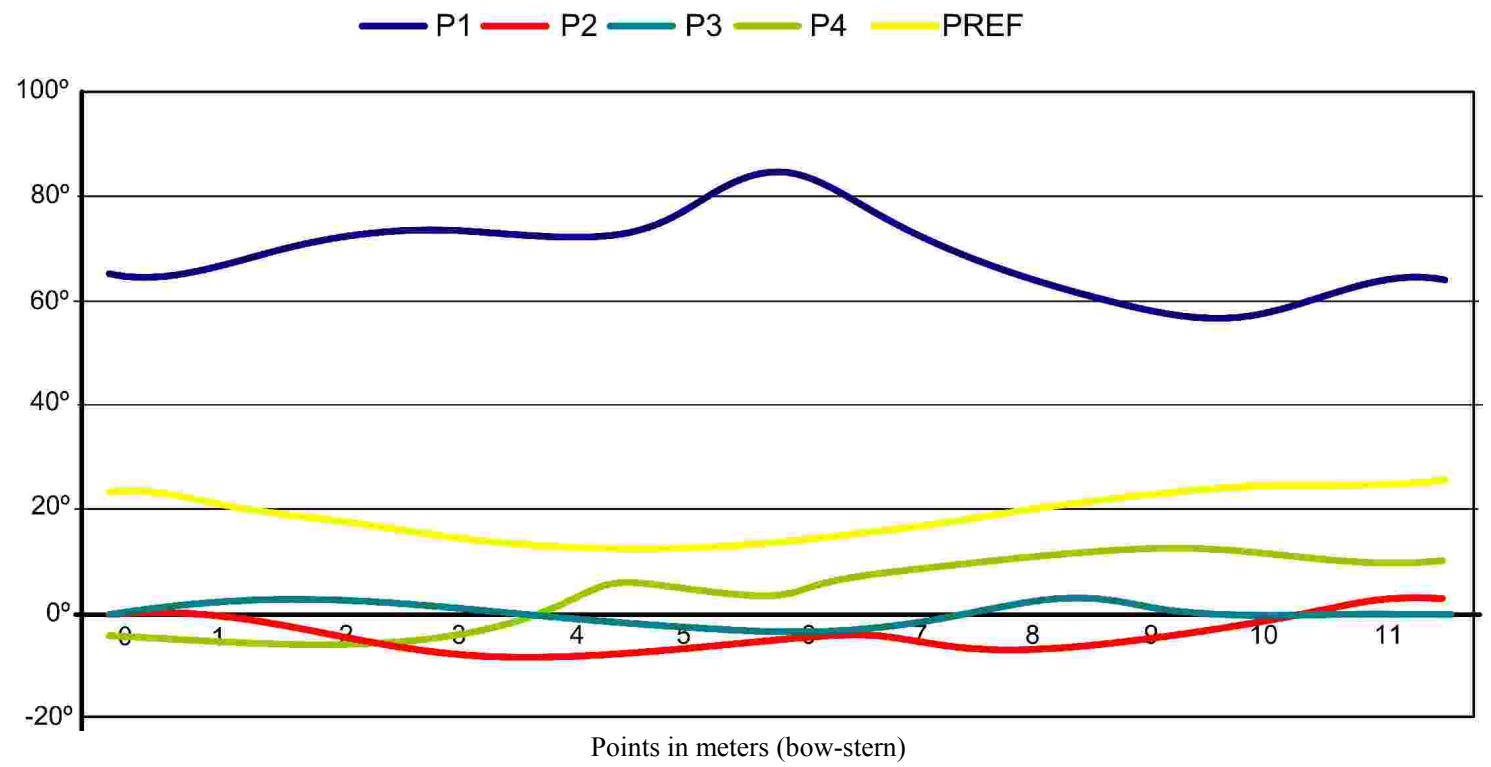

Figure 7. Absolute declination of the horizontal magnetic field along rows inside zone $Z_{3}$.

sources, as shown in Figure 9 (yellow color). Each coil is used to compensate one of the identified magnetic sources.

Using the optimization technique presented in [1], we can compute the values of the currents and the number of turns in each coil needed to reduce the magnetic field created by the identified sources. In this case, the optimization algorithm converged for a current of -2000 Amp/Turn in coil MBr and $7000 \mathrm{Amp} /$ Turn for coil MEr. In Figure 10 we show the $x$-component of the magnetic field obtained after compensation, indicating a reduction of the magnetic field in about $71 \%$ with respect to the initial values shown in Figure 8.

The compensation of the type 2 anomaly present in platform (P4) is more complex, since a strong source causing the magnetic anomaly cannot be identified. This magnetic anomaly is probably due to the interaction of several weak sources distributed in several areas of the platform. Figure 11 shows the measured $x$-component of the magnetic field in platform (P4) before compensation.

In this case, compensation is performed in the area where the sensitive sensors must operate (in our case between columns 12 and 25). For this purpose a total of fifteen coils have been used, distributed in the relevant area 
of the deck. In Figure 12 we show a drawing of the deck, indicating in red the zone of the magnetic anomaly. The numbers in blue show the location of the fifteen coils used for compensation.

Using again the method described in [1], the currents to each coil and the number of turns are optimized. This information is collected in Table 4.

To show the effectiveness of the proposed approach, we present in Figure 13 the measured magnetic field

Table 3. Final values obtained for GMERPR and GMERPC.

\begin{tabular}{|c|c|c|c|c|}
\hline & & \multicolumn{3}{|c|}{ Anomaly } \\
\hline Platform & GMERPR & $\begin{array}{c}\mathrm{NO} \\
\text { GMERPF } \\
<13\end{array}$ & $\begin{array}{c}\text { TYPE } 2 \\
13< \\
G M E R P F< \\
18\end{array}$ & $\begin{array}{c}\text { TYPE } 1 \\
(G M E R P F \\
>18)\end{array}$ \\
\hline PREF & 11 & & & \\
\hline $\mathrm{P} 1$ & 20 & & & $X$ \\
\hline $\mathrm{P} 2$ & 10 & $\mathrm{X}$ & & \\
\hline P3 & 6 & $\mathrm{X}$ & & \\
\hline \multirow[t]{2}{*}{$\mathrm{P} 4$} & 15 & & $\mathrm{X}$ & \\
\hline & & & Anomaly & \\
\hline Platform & GMERPC & $\begin{array}{c}\text { NO } \\
\text { GMERPC } \\
<7\end{array}$ & $\begin{array}{c}\text { TYPE } 2 \\
7< \\
\text { GMERPC } \\
<10\end{array}$ & $\begin{array}{c}\text { TYPE } 1 \\
(G M E R P C \\
>10)\end{array}$ \\
\hline PREF & 4 & & & \\
\hline $\mathrm{P} 1$ & 12 & & & $\mathrm{X}$ \\
\hline $\mathrm{P} 2$ & 6 & $X$ & & \\
\hline P3 & 4 & $X$ & & \\
\hline P4 & 9 & & $\mathrm{X}$ & \\
\hline
\end{tabular}

obtained after compensation. Results demonstrate that a gradient reduction of $30 \%$ can be achieved, which is sufficient for most practical applications.

In Table 5 values for parameters $D M P R X, G E P R X$, GMERPR and GMERPC before and after compensation are shown.

It can be observed from above table that substantial reduction in the anomaly parameters are obtained after applying the compensation techniques. In particular, the new values obtained are below the specified thresholds given in Table 1, indicating that the platforms are now free of anomalies, thus fully validating the approach presented in this paper.

\section{Conclusions}

Sometimes naval platforms present local magnetic anomalies that cannot be compensated by standard degaussing systems. The authors propose in this paper a novel method to detect, quantify and correct these local perturbations. This will avoid the application of complex and expensive deperming processes.

The detected anomalies have been classified in two types depending on the number of sources and the magnetization strength. The anomaly of type 1 is a strong anomaly with well identified source. The anomaly of type 2 is a medium anomaly produced by several weak sources (several elements) of the naval platform. Results show that the compensation technique is more effective and easy to introduce in the type 1 anomaly.

The described method has been applied to a real situation composed of five naval platforms. The technique has proved its effectiveness in anomaly detection, quantification and subsequent compensation of undesired local magnetic anomalies, present in the investigated platforms.

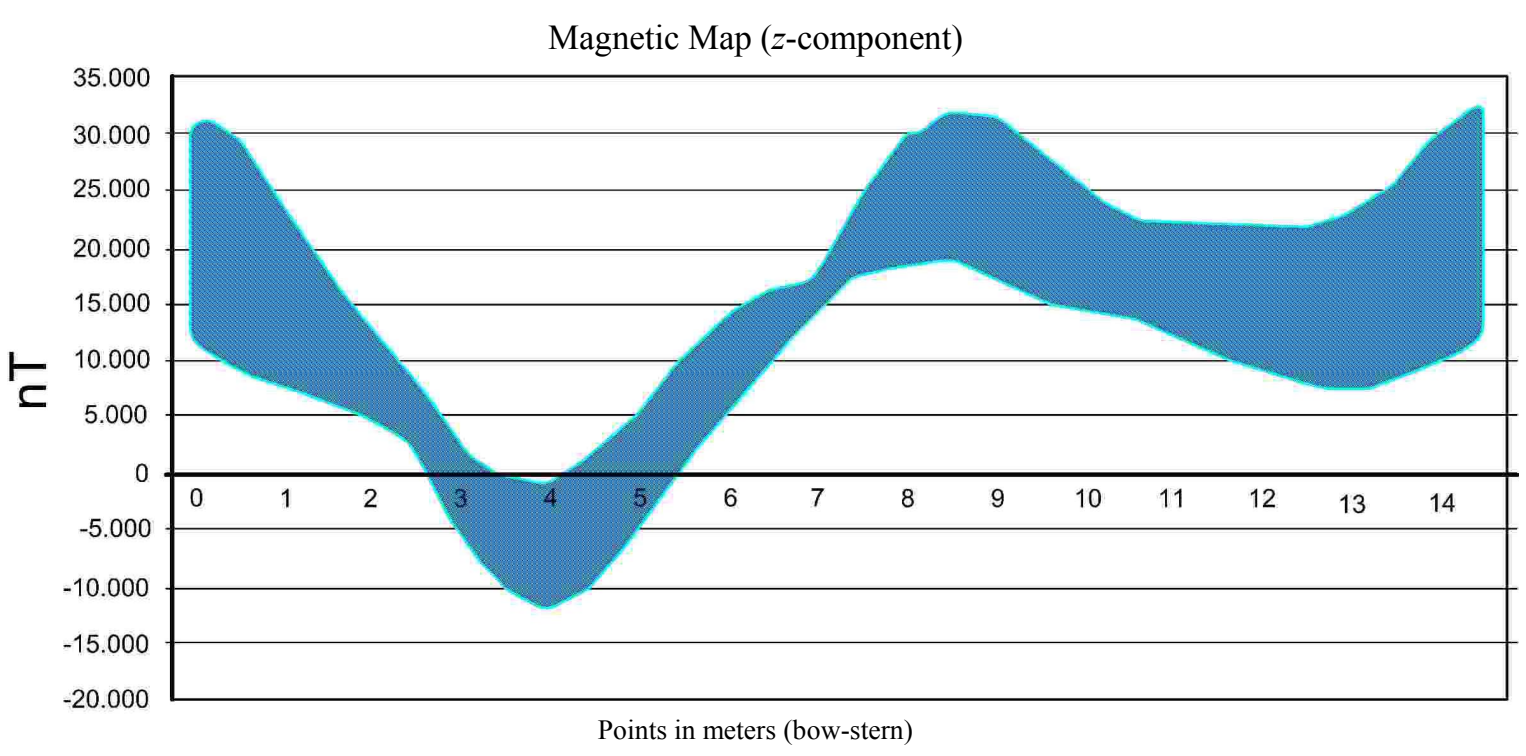

Figure 8. Measured $x$-component of the magnetic field in platform (P1) before compensation, from port to starboard. 


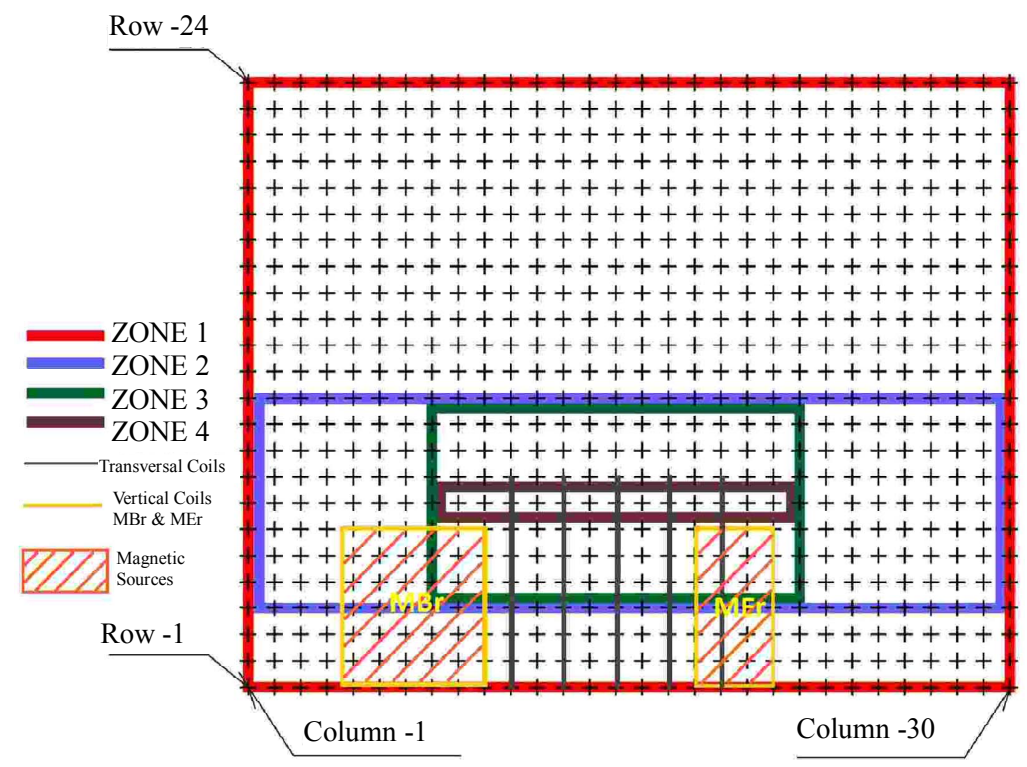

Figure 9. Surface of the deck showing the mesh. The location of the additional coils used in the compensation of platform (P1) is marked with yellow lines.

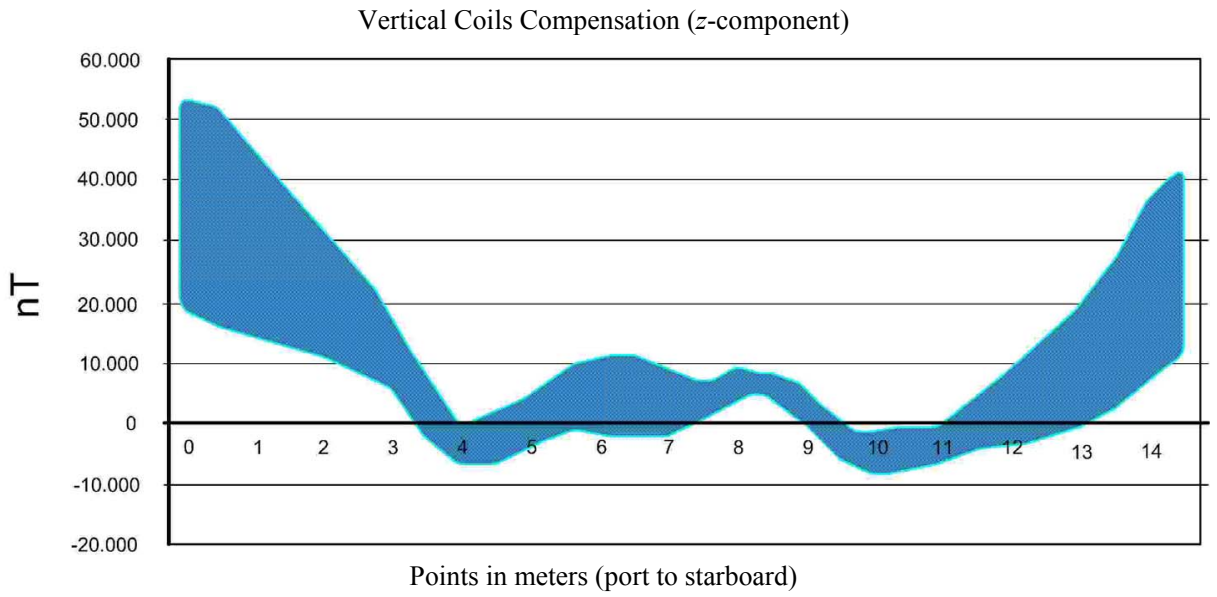

Figure 10. Measured $x$-component of the magnetic field for rows 2 - 10 from port to starboard after compensation with coils MBr and MEr.

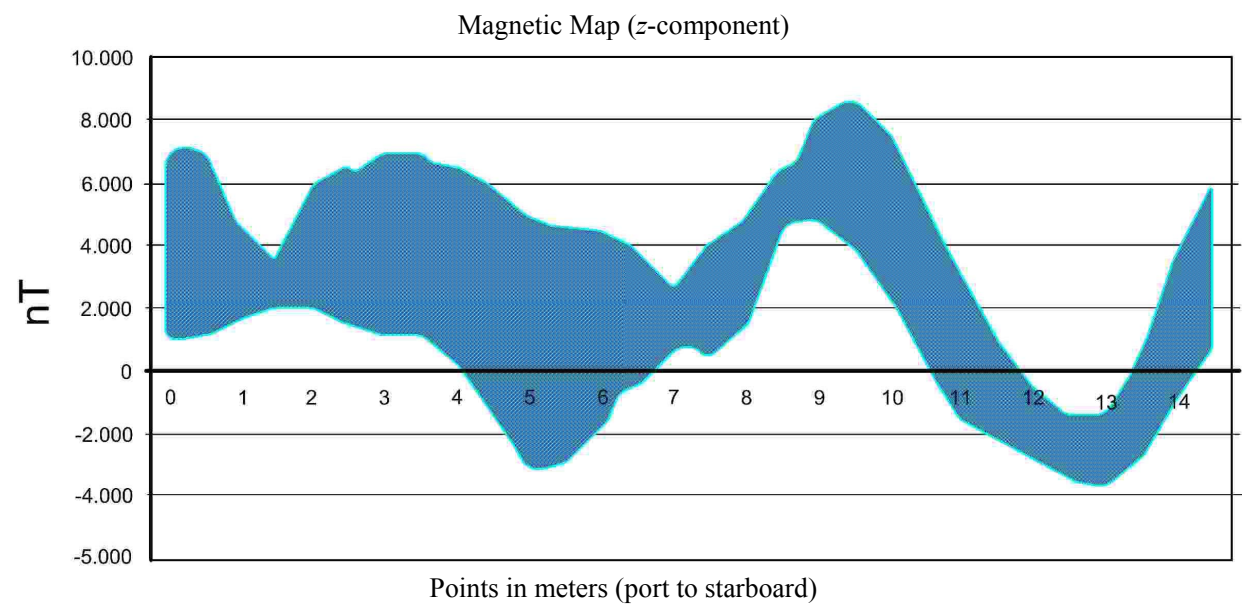

Figure 11. Measured $x$-component of the magnetic field in platform (P4) before compensation, from port to starboard. 


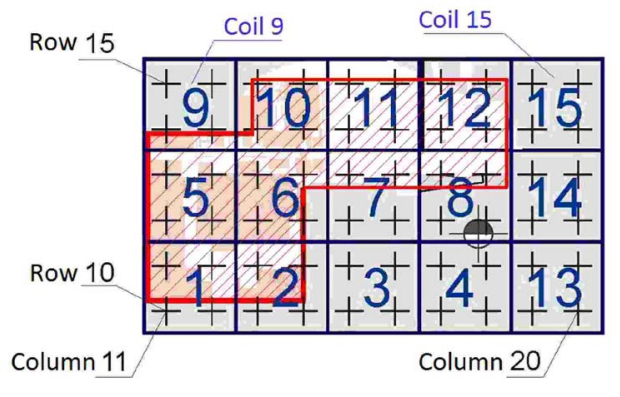

Figure 12. Zone of the magnetic anomaly (in red), and distribution of the coils for compensation.
Table 4. Optimized values of the currents to each coil needed for compensation of platform (P4).

\begin{tabular}{cccccc}
\hline Coil & Amp/Turn & Coil & Amp/Turn & Coil & Amp/Turn \\
\hline 1 & 136 & 6 & 138 & 11 & 67 \\
2 & 128 & 7 & 334 & 12 & 450 \\
3 & 48 & 8 & 475 & 13 & 433 \\
4 & 274 & 9 & 97 & 14 & 292 \\
5 & 99 & 10 & 152 & 15 & 142 \\
\hline
\end{tabular}

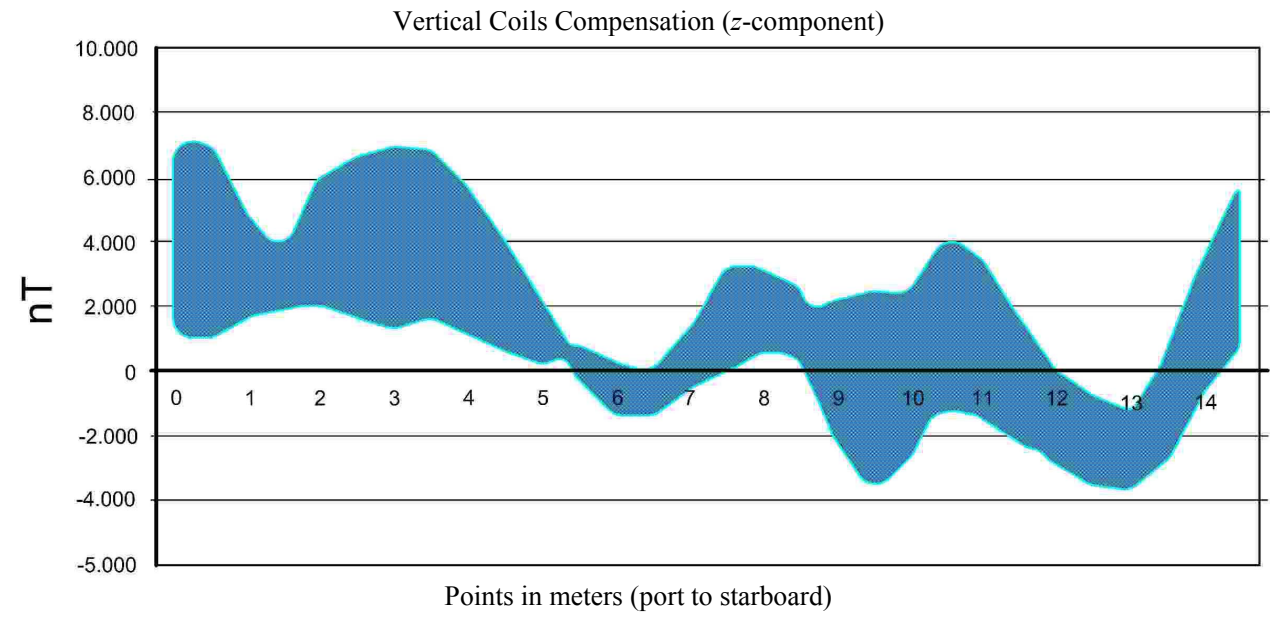

Figure 13. Measured $x$-component of the magnetic field from port to starboard after compensation with 15 degaussing coils.

Table 5. Values obtained for DMPRX, GEPRX, GMERPR and GMERPC before and after compensation for Platforms P1 and P4.

\begin{tabular}{rccc}
\hline Platform & DMPRX/REF & GEPRX/REF & GMERPR/PC \% \\
\hline P1 Before & 3.98 & 3.88 & $20 / 12$ \\
P1 After & 0.57 & 1.05 & $5 / 6$ \\
P4 Before & 1.21 & 1.98 & $15 / 9$ \\
P4 After & 0,82 & 1.11 & $4 / 4$ \\
\hline
\end{tabular}

\section{Acknowledgements}

This work was supported under Spanish National project TEC2010-21520-C04-04 and European Feder Fundings.

\section{REFERENCES}

[1] A. V. Madrid and A. Á. Melcón, "Using Genetic Algorithms for Compensating the Local Magnetic Perturbation of a Ship in the Earth's Magnetic Field," Microwave and Optical Technology Letters, Vol. 47, No. 3, 2005, pp. 281287.

[2] F. L. Dorze, J. P. Bongiraud, J. L. Coulomb, P. Labie and X. Brunote, "Modeling of Degaussing Coils Effects in
Ships by the Method of Reduced Scalar Potencial Jump," IEEE Transaction on Magnetics, Vol. 34, No. 5, 1998, pp. 2477-2480.

[3] X. Brunotte and G. Meunier, "Line Element for Efficient Computation of the Magnetic Field Created by Thin Iron Plates," IEEE Transaction on Magnetics, Vol. 26, No. 5, 1990, pp. 2196-2198.

[4] X. Brunotte, G. Meunier and J. P. Bongiraud, "Ship Magnetizations Modelling by the Finite Element Method," IEEE Transaction on Magnetics, Vol. 29, No. 2, 1993, pp. 1970-1975.

[5] D. Rogers, P. J. Leonard and H. C. Lai, "Surface Elements for Modelling 3D Fields around Thin Iron Sheets," IEEE Transaction on Magnetics, Vol. 29, No. 25, 1993, pp. 1483-1486.

[6] J. J. Holmes, "Application of Models in the Design of Underwater Electromagnetic Signature Reduction Systems," Naval Engineers Journal, Vol. 119, No. 4, 2007, pp. 19-29. doi:10.1111/j.1559-3584.2007.00083.x

[7] D. S. Weile and E. Michielssen, "Genetic Algorithm Optimization Applied to Electromagnetics: A Review," IEEE Transaction on Antennas and Propagation, Vol. 45, No. 3, 1997, pp. 343-353.

[8] D. E. Goldberg, "Genetic Algorithm in Search, Optimization and Machine Learning," Addison-Wesley Publishing Company, Boston, 1989. 
[9] H. Liu and Z. Ma, "Optimization of Warship Degaussing System Based on Poly-Population Particle Swarm Algorithm," International Conference on Mechatronic and Automation, ICMA, Harbin, 5-8 August 2007, pp. 31333137.

[10] W. C. Potts, "The Magnetic Field of a Ship and Its Neutralization by Coil Degaussing," General Journal of the
Institution of Electrical Engineers-Part I, Vol. 93, No. 71, 1946, pp. 488-499.

[11] Y.-T. Yang, Z.-Y. Shi, J.-G. Lu and Z.-Z. Guan, "A Magnetic Disturbance Compensation Method Based on Magnetic Dipole Magnetic Field Distributing Theory," Journal of China Ordenance, Vol. 5, No. 3, 2009, pp. 185-191. 